\title{
Boundary Control by Boundary Observer for Hyper-redundant Robots
}

\author{
M. Ivanescu, D. Cojocaru, N. Bizdoaca, M. Florescu \\ N. Popescu, D. Popescu, S. Dumitru
}

\author{
Mircea Ivanescu, Dorian Cojocaru, \\ Nicu Bizdoaca, Mihaela Florescu, Sorin Dumitru \\ Mechatronic Department, University of Craiova \\ E-mail: ivanescu, cojocaru, nicu, mihaela, dumitru@robotics.ucv.ro
}

\section{Nirvana Popescu, Decebal Popescu}

Department of Computer Science,

Politehnica University Bucharest,

E-mail: nirvana.popescu, decebal.popescu@cs.pub.ro

\begin{abstract}
The control problem of a class of hyper-redundant arms with continuum elements, with boundary measuring and control is discussed. First, the dynamic model of the continuum arm is presented. The measuring systems are based on the film sensors that are placed at the terminal sub-regions of the arm. The observers are proposed in order to reconstruct the full state of the arm. A back-stepping method is used to design a boundary control algorithm. Numerical simulations of the arm motion toward an imposed position are presented. An experimental platform shows the effectiveness of the proposed methods.
\end{abstract}

Keywords: hyper-redundant system, distributed parameter system, observer, control.

\section{Introduction}

The hyper-redundant arms are a class of manipulators that can achieve any position and orientation in space. A special class of these robots is represented by the mechanical structures with continuum elements described by distributed parameter model. The control of these systems is very complex and a great number of researchers have tried to offer solutions. In $[2,3]$, Gravagne analyzed the kinematic models. Important results were obtained by Chirikjian and Burdick [4], which laid the foundations for the kinematical theory of hyper-redundant robots. Their results are based on a "backbone curve" that captures the robot's macroscopic geometric features. Mochiyama has also investigated the problem of controlling the shape of an HDOF rigid - link robot with two-degree-of-freedom joints using spatial curves [5]. In other papers [6,7], several technological solutions for actuators used in hyper-redundant structures are presented and conventional control systems are introduced. In [8] control problem of a class that performs the grasping function by coiling is discussed. A frequential stability criterion for the grasping control problem is proposed in [9].

In this paper, control problem of a class of hyper-redundant arms with continuum elements, with boundary measuring and control is discussed. The development of feedback controllers and compensators for these models is a very complex problem. The difficulty is determined by the complexity of the dynamic models expressed by partial differential equations and by the observability problems in distributed parameter systems. An essential part of designing feedback controllers for these models is designing practical controllers that are implementable. Standard feedback control design assumes full-state feedback with measurements of the entire state. Recent 
advances in distributed sensor technology, as Polyvinylidene Fluoride film sensors [10], allow ensuring a good quality of position measuring in distributed systems. However, the use of these sensors on all surface of continuum arms, is not practical due to mechanical constraints. In fact, the sensors are placed on the boundary of the elements. In this case, the development of the state-feedback controllers needs to design state observers. The observability problems are solved by an approach derived from the Luenberger observer type and the "back-stepping method" developed in [11].

The paper is organized as follows: section II presents technological and theoretical preliminaries, section III studies the dynamic model, section IV presents the control by boundary observer, section $\mathrm{V}$ verifies the control laws by computer simulation and section VI presents an experimental model.

\section{Technological and Theoretical Preliminaries}

The hyper-redundant technological models are complex structures that operate in 3D space, but the control laws of the elements can be infer from the planar models. For this reason, the model discussed in this paper is a $2 \mathrm{D}$ model.

The technological model basis is presented in Fig.1. It consists of a number $(\mathrm{N})$ of continuum segments, each segment having a layer structure that ensures the flexibility, the driving and position measuring (Fig.2).

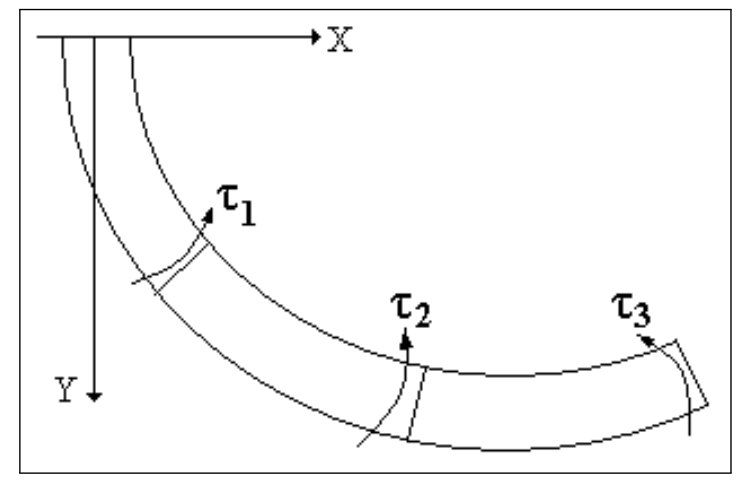

Figure 1:

The high flexibility is obtained by an elastic non-extensible backbone rod with distributed damping and negligible shear effects.

The driving system consists by two antagonistic cable actuators that are connected at the end of each segment and determine the bending of the arm.

The position measuring of the segment is obtained by an electro-active polymer curvature sensor that is placed on the surface at the terminal sub-regions of each segment. These sensors can measure the curvature on the boundary of the segment $(\mathrm{s}=0$ or $\mathrm{s}=\mathrm{l})$. The essence of the segment $i$ is the backbone curve $C_{i}$. The length of each segment is 1 . The independent parameter $\mathrm{s}$ is related to the arc-length from origin of the curve $C_{i}, s \in \Omega, \Omega=[0, l]$. The curvature of the segment is [13] (Fig.3)

where

$$
\chi=\frac{d \phi}{d s}
$$

$$
\phi=\frac{s}{R_{c}}
$$




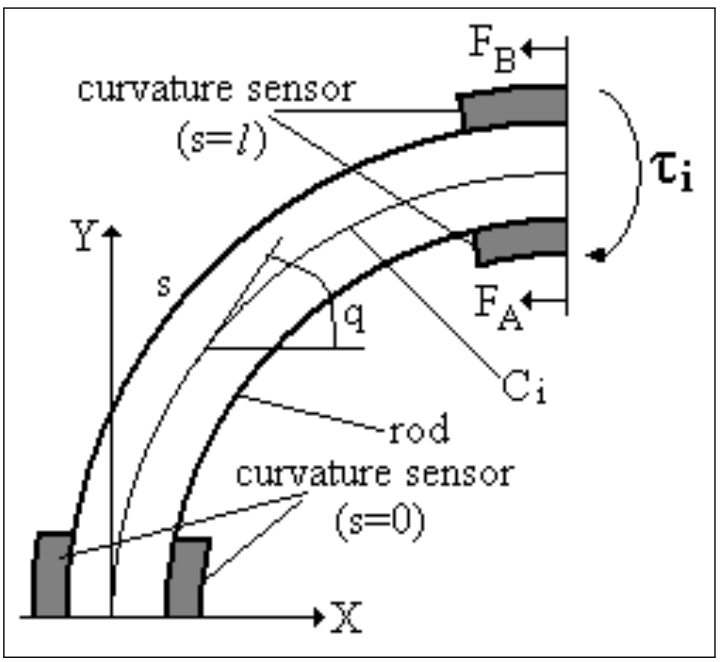

Figure 2:

represents the angle of the current position and $R_{C}$ is the radix of the arc. We denote by $\tau$ the equivalent moment at the end of the segment $(\mathrm{s}=1)$ exercised by the cable forces $F_{A}$ and $F_{B}$. The position of a point $s$ on curve $C_{i}$ is defined by the position vector $r=r(s), s \in[0, l]$ - For a dynamic motion, the time variable will be introduced, $r=r(s, t)$. The segment has the elastic modules E, the moment of inertia I, the bending stiffness EI, the linear mass density $\rho$ and rotational inertial density $\mathrm{I}_{\rho}$.

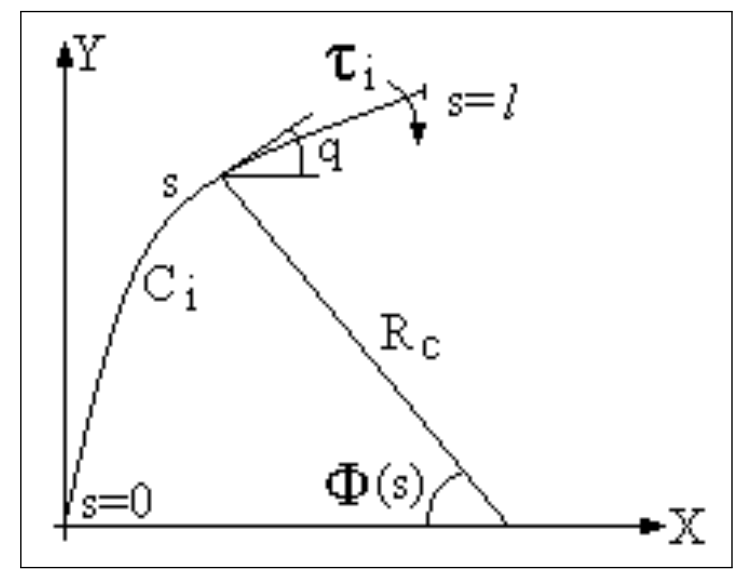

Figure 3:

\section{Dynamic model}

The dynamic model of a segment can be derived from the Hamiltonian principle. Using the same procedure as in[3] yields the partial differential equations of the arm segment,

$$
I_{\rho} \ddot{q}+b_{1} \dot{q}-E I q_{s s}+c_{1} q=0
$$

with the initial and boundary conditions

$$
\dot{\mathrm{q}}(0, s)=0
$$




$$
\begin{gathered}
\operatorname{EIq}_{s}(t, l)=\tau \\
\mathrm{q}_{s}(t, 0)=0
\end{gathered}
$$

where $\mathrm{q}=\mathrm{q}(\mathrm{t}, \mathrm{s}), \dot{\mathrm{q}}, \mathrm{q}_{\mathrm{s}}, \mathrm{q}_{\mathrm{ss}}$ denote $\frac{\partial \mathrm{q}(\mathrm{t}, \mathrm{s})}{\partial \mathrm{t}}, \frac{\partial \mathrm{q}(\mathrm{t}, \mathrm{s})}{\partial \mathrm{s}}, \frac{\partial^{2} \mathrm{q}(\mathrm{t}, \mathrm{s})}{\partial \mathrm{s}^{2}}$, respectively, $\mathrm{b}_{1}$ is the equivalent damping coefficient and $c_{1}$ characterizes the elastic behavior.

The equations (3.1) - (3.4) can be rewritten as:

$$
\begin{gathered}
\ddot{\mathrm{q}}=\mathrm{aq}_{\mathrm{ss}}+\mathrm{b} \dot{\mathrm{q}}+\mathrm{cq} \\
\mathrm{q}_{\mathrm{s}}(\mathrm{t}, 0)=0 \\
\mathrm{q}_{\mathrm{s}}(\mathrm{t}, \mathrm{l})=\mathrm{d} \cdot \tau \\
\dot{\mathrm{q}}(0, \mathrm{~s})=0, s \in[0, \mathrm{l}]
\end{gathered}
$$

where

$$
\mathrm{a}=\frac{\mathrm{EI}}{\mathrm{I}_{\rho}} ; \mathrm{b}=-\frac{\mathrm{b}_{1}}{\mathrm{I}_{\rho}} ; \mathrm{c}=-\frac{\mathrm{c}_{\mathrm{i}}}{\mathrm{I}_{\rho}} ; \mathrm{d}=\frac{1}{\mathrm{EI}}
$$

The input of the system is represented by the moment $\tau$ applied at the boundary $s=1$ of the arm. The output is determined by the angle values measured by the sensor,

$$
y(t)=q(0, t)
$$

or

$$
y(t)=q(l, t)
$$

\section{Control by boundary observer}

We shall analyze two cases: 1) the measurement system allows to measure the angle at the bottom end $\mathrm{s}=\mathrm{O} ; 2$ ) the measurement system allows to measure the angle at the upper end $\mathrm{s}=\mathrm{l}$. For the both cases, a regional boundary observer is introduced in order to reconstruct the state in the domain and generate a full-state feedback.

\subsection{Problem 1: $\mathrm{q}(\mathrm{t}, 0)$ is available for measurement (Fig.4)}

The following observer is proposed:

$$
\begin{gathered}
\ddot{\hat{\mathrm{q}}}=a \hat{\mathrm{q}}_{s s}+b \dot{\hat{\mathrm{q}}}+c \hat{\mathrm{q}}+\mathrm{k}_{1}(\mathrm{~s})(\mathrm{q}(\mathrm{t}, 0)-\hat{\mathrm{q}}(\mathrm{t}, 0)) \\
\hat{\mathrm{q}}_{s}(t, 0)=k_{0}(\mathrm{q}(\mathrm{t}, 0)-\hat{\mathrm{q}}(\mathrm{t}, 0)) \\
\hat{\mathrm{q}}_{s}(t, l)=\mathrm{d} \cdot \tau
\end{gathered}
$$




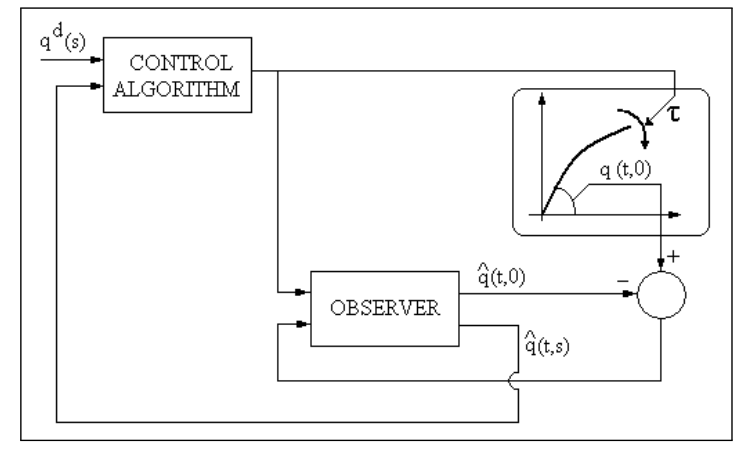

Figure 4:

$$
\dot{\hat{\mathrm{q}}}(0, \mathrm{l})=0
$$

where $\hat{\mathrm{q}}=\hat{\mathrm{q}}(\mathrm{t}, \mathrm{s})$ is the observer state and $\mathrm{k}_{1}(\mathrm{~s}), \mathrm{k}_{\mathrm{o}}$ are a function and a constant, respectively, that define the observer parameters. The objective is to determine these parameters in order to reconstruct the state in the domain, i.e., to find $k_{1}(s)$ and $k_{0}$ such that $\hat{q}$ converges to $q$ as time goes to infinity.

An error variable $\tilde{q}$ is introduced

$$
\tilde{\mathrm{q}}=\mathrm{q}-\hat{\mathrm{q}}
$$

and the error system will be:

$$
\begin{gathered}
\ddot{\tilde{\mathrm{q}}}=a \tilde{\mathrm{q}}_{s s}+b \dot{\tilde{\mathrm{q}}}+c \tilde{\mathrm{q}}-k_{1}(s) \tilde{\mathrm{q}}(t, 0) \\
\tilde{\mathrm{q}}_{s}(t, 0)=-k_{0} \tilde{\mathrm{q}}(\mathrm{t}, 0) \\
\tilde{\mathrm{q}}_{s}(t, l)=0 \\
\dot{\tilde{q}}(0, l)=0
\end{gathered}
$$

where

$$
\lim _{t \rightarrow \infty} \tilde{q}(t, s)=0, s \in[0, l]
$$

We consider that the desired states of the arm motion are given by the curve $C_{d}$,

$$
C_{d}:\left(q^{d}(s) . s \in[0, l]\right)
$$

The control problem is to find the moment control law $\tau$ in order to achieve the desired state.

Control algorithm 1. The closed loop control law of the arm (3.5) - (3.8) with the boundary observer (4.1) - (4.4) is given by

$$
\tau(t)=E I\left(-k(l, l)\left(\widehat{q}(t, l)-q_{d}(l)\right)+q_{s}^{d}(l)-\int_{0}^{l} k_{s}(l, z)\left(\widehat{q}(t, z)-q^{d}(z)\right) d z\right)
$$

where $\mathrm{k}(\mathrm{s}, \mathrm{z})$ is the solution of the following partial differential equations, 


$$
\begin{gathered}
-a k_{s s}(s, z)+a k_{z z}(s, z)+c k(s, z)=0 \\
k_{s s}(s, s)=\frac{c}{2 a}(l-s)
\end{gathered}
$$

with the boundary condition

$$
k(l, z)=0, z \in[0, l]
$$

and the observer parameters are defined by the equations

$$
\begin{gathered}
k_{0}=-k(0,0) \\
k_{1}(s)=-a k_{z}(s, 0)-\int_{0}^{s} k_{1}(z) k(s, z) d z
\end{gathered}
$$

Proof. See Appendix 1.

\subsection{Problem 2: $\mathrm{q}(\mathrm{t}, \mathrm{l})$ is available for measurement (Fig.5)}

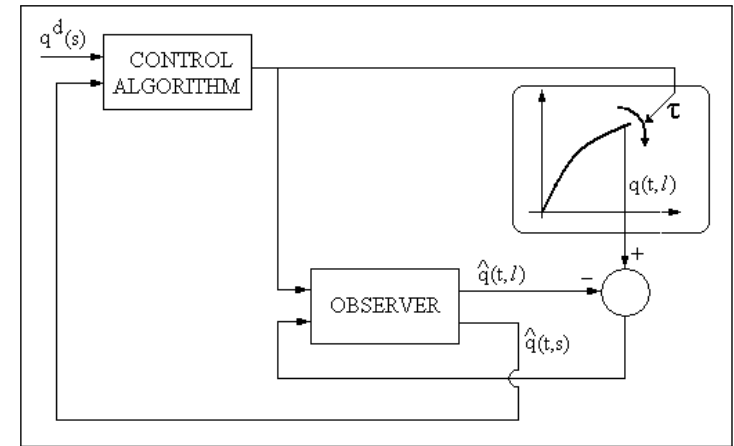

Figure 5:

The observer will be

$$
\ddot{\hat{q}}=a \hat{q}_{s s}+b \dot{\hat{q}}+c \hat{q}+k_{1}(s)(q(t, l)-\hat{q}(t, l))
$$

with the boundary conditions

$$
\begin{gathered}
\hat{\mathrm{q}}_{s}(t, 0)=\hat{\mathrm{q}}_{0} \\
\hat{\mathrm{q}}_{s}(t, l)=\mathrm{k}_{0}(\mathrm{q}(\mathrm{t}, \mathrm{l})-\hat{\mathrm{q}}(\mathrm{t}, \mathrm{l}))+\mathrm{d} \tau \\
\dot{\hat{\mathrm{q}}}(0,0)=0
\end{gathered}
$$

The error system has the form

$$
\begin{gathered}
\ddot{\tilde{\mathrm{q}}}=\mathrm{a} \tilde{\mathrm{q}}_{s s}+\mathrm{b} \dot{\tilde{\mathrm{q}}}+\mathrm{c} \tilde{\mathrm{q}}-\mathrm{k}_{1}(\mathrm{~s}) \tilde{\mathrm{q}}(\mathrm{t}, \mathrm{l}) \\
\tilde{\mathrm{q}}_{s}(\mathrm{t}, 0)=-\hat{\mathrm{q}}_{0}
\end{gathered}
$$




$$
\begin{gathered}
\tilde{\mathrm{q}}_{s}(\mathrm{t}, \mathrm{l})=-\mathrm{k}_{0} \tilde{\mathrm{q}}(\mathrm{t}, \mathrm{l}) \\
\dot{\tilde{\mathrm{q}}}(0,0)=0
\end{gathered}
$$

Control algorithm 2. The closed loop control law of the arm (3.5) - (3.8) with the boundary observer (4.17) - (4.20) is given by

$$
\tau(t)=E I\left(q_{s}^{d}(l)-k(l, l)\left(q^{d}(l)-\hat{q}(t, l)\right)\right)
$$

where $\mathrm{k}(\mathrm{s}, \mathrm{z})$ is the solution of the following equations

$$
\begin{gathered}
\mathrm{ak}_{s s}(\mathrm{~s}, z)-\mathrm{ak}_{z z}(\mathrm{~s}, z)+\mathrm{ck}(\mathrm{s}, z)=0 \\
\mathrm{k}(\mathrm{s}, \mathrm{s})=\frac{\mathrm{c}}{2 \mathrm{a}} \mathrm{s}
\end{gathered}
$$

with the boundary conditions

$$
k(0, z)=0, z \in[0, l]
$$

The observer parameters are obtained by solving the following equations

$$
\begin{gathered}
k_{0}=k(l, l) \\
k_{1}(s)=a k_{s}(s, l)+\int_{s}^{l} k_{1}(z) k(s, z) d z
\end{gathered}
$$

Proof. See Appendix 2.

\section{Simulation}

A hyper-redundant manipulator control with continuum segments is simulated. The parameters of the arm were selected as: bending stiffness $\mathrm{EI}=1$, rotational inertial density $\mathrm{I}_{\rho}=$ $0.001 \mathrm{~kg} \cdot \mathrm{m}^{2}$, damping ratio 0.35 and elastic coefficient 4.8 . These constants are realistic for long thin backbone structures. The length of each segment is $l=1$.

Problem 1. $\mathrm{q}(\mathrm{t}, 0)$ is available for measurement

The observer parameters $k_{0}, k_{1}(s)$ are computed. First, a numerical solution for $k(s, z)$ is obtained by the integration of partial differential equation (4.13) with boundary conditions (4.14) and (4.15). The result is presented in Fig.6.

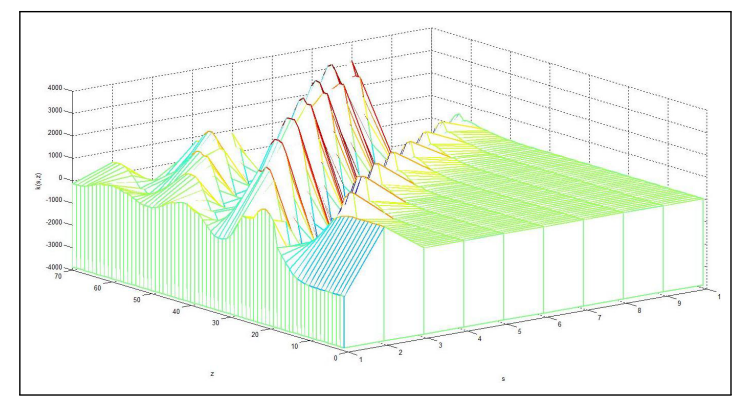

Figure 6: 
The parameter $k_{0}$ is obtained from (4.16) as $k_{0}=-\mathrm{k}(0,0)=12.4$

The parameter $k_{1}$ is determined from the integral equation (4.17). The numerical solution is presented in the Table 1.

Table 1. Parameter $k_{1}(s)$ for the Problem 1

\begin{tabular}{|c|c|c|c|c|c|c|}
\hline $\mathrm{S}$ & 0 & 0.2 & 0.4 & 0.6 & 0.8 & 1.0 \\
\hline $\mathrm{k}_{1}(\mathrm{~s})$ & -3.30 & -3.80 & -0.52 & 0.14 & 0.06 & 0.00 \\
\hline
\end{tabular}

A desired trajectory defined as

$$
q^{d}(s)=\pi s^{2}, s \in[0,1]
$$

is proposed and the control law (4.12) is used A MATLAB simulation of the observer arm system is implemented. The result is presented in Fig.7.

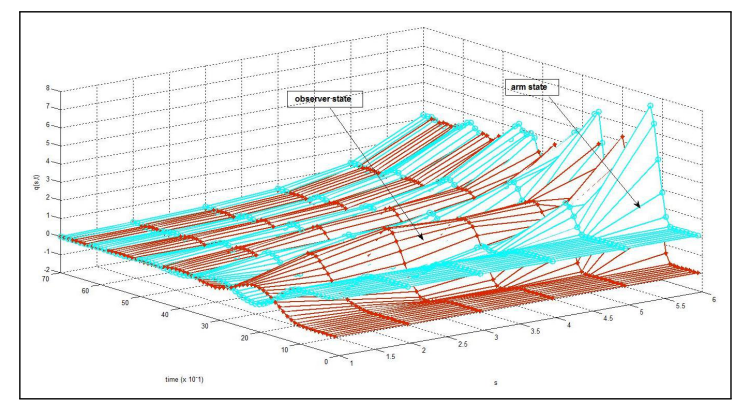

Figure 7:

We can remark the convergence of the estimated state of the observer to the system state and the quality of evolution on the trajectory to the desired state.

Problem 2. $\mathrm{q}(\mathrm{t}, \mathrm{l})$ is available for measurement

The numerical solution of $\mathrm{k}(\mathrm{s}, \mathrm{z})$ is obtained from the equation (4.28) with the boundary conditions (4.28), (4.29). The result is presented in Fig.8.

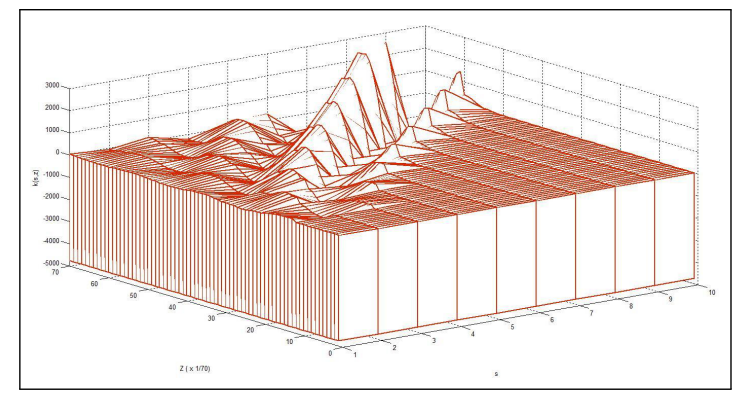

Figure 8:

The observer parameters are obtained from (4.30), (4.31), $\mathrm{k}_{0}=\mathrm{k}(1,1)=12.4$ and $\mathrm{k}_{1}(\mathrm{~s})$ is represented in Table 2.

Table 2. $k_{1}(\mathrm{~s})$ for the Problem 2

\begin{tabular}{|c|c|c|c|c|c|}
\hline $\mathrm{S}$ & 0 & 0.2 & 0.4 & 0.6 & 1.0 \\
\hline $\mathrm{k}_{1}(\mathrm{~s})$ & 4.83 & 8.64 & 2.15 & -0.01 & 0.00 \\
\hline
\end{tabular}

A desired trajectory 


$$
q^{d}(s)=1.4 s^{2}, s \in[0,1]
$$

is proposed.

The control law (4.26) is applied and a simulation in MATLAB of the global system, as presented in Fig.5, is implemented. The result is shown in Fig.9.

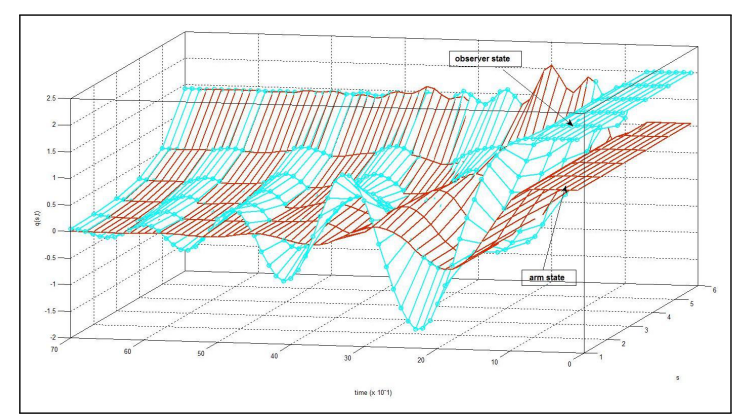

Figure 9:

\section{Experimental results}

In order to verify the suitability of the control algorithm, a platform with a 3D hyperredundant arm has been employed for testing. The arm consists of three continuum segments with a flexible backbone rod. Three antagonistic cable actuators for each segment ensure the actuation system (Fig.10, Fig.11). The force in each cable is determined by the DC motors and a transmission system.

e
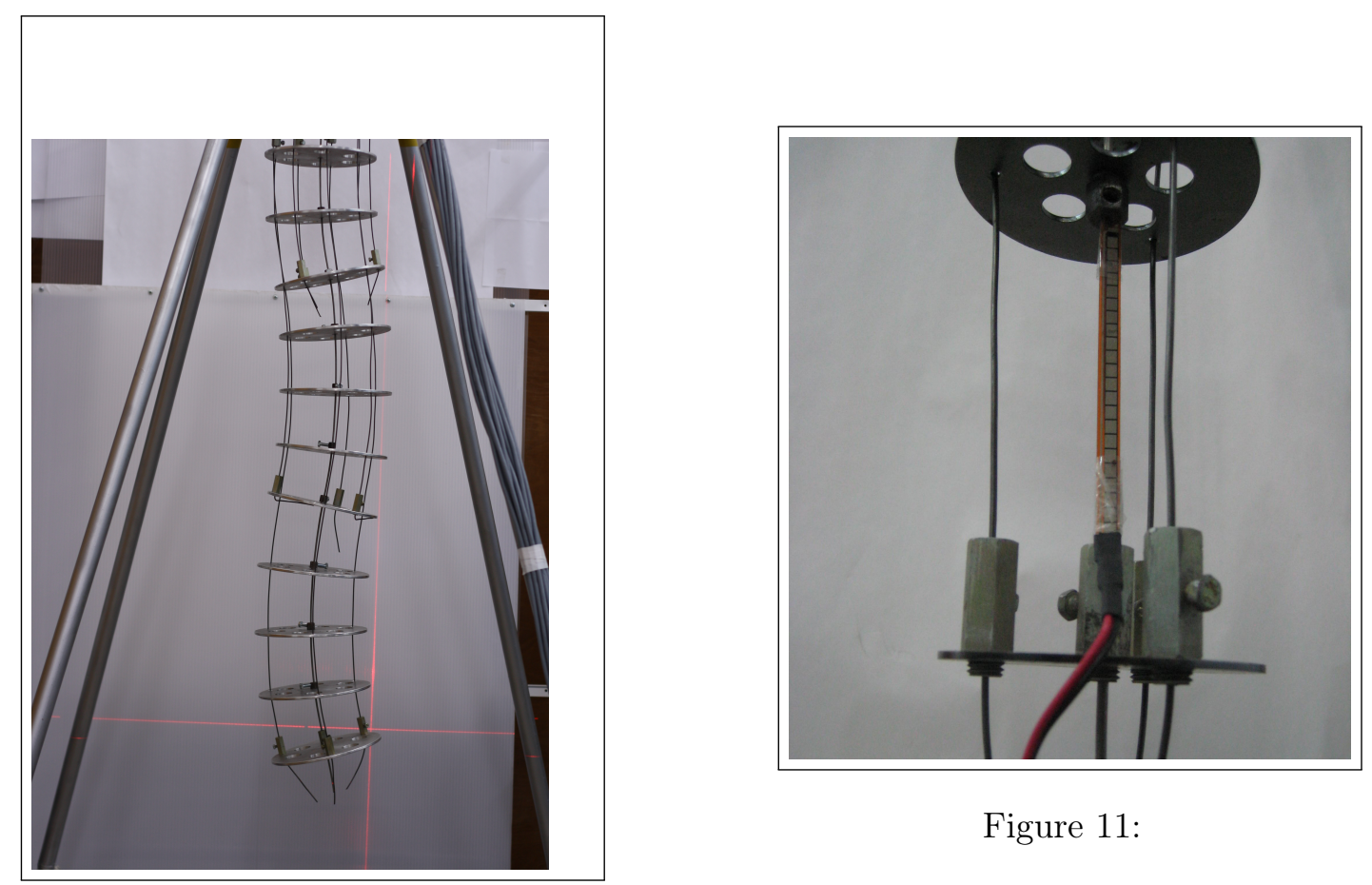

Figure 11:

Figure 10: 
A polymer thick film layer is placed on the upper level of the rod on each segment ( $\mathrm{s}=\mathrm{l}=0.3 \mathrm{~m}$ ). A sensor exhibits a decrease in resistance when an increase of the film curvature is used. A Wheatstone bridge system is used to measure the variation of the resistance. A Quancer based platform is used for control and signal acquisition. A control law (4.27) with $\mathrm{q}_{\mathrm{d}}(\mathrm{s})=40 \mathrm{~s}^{2}$ is implemented. The result is presented in Fig.12.

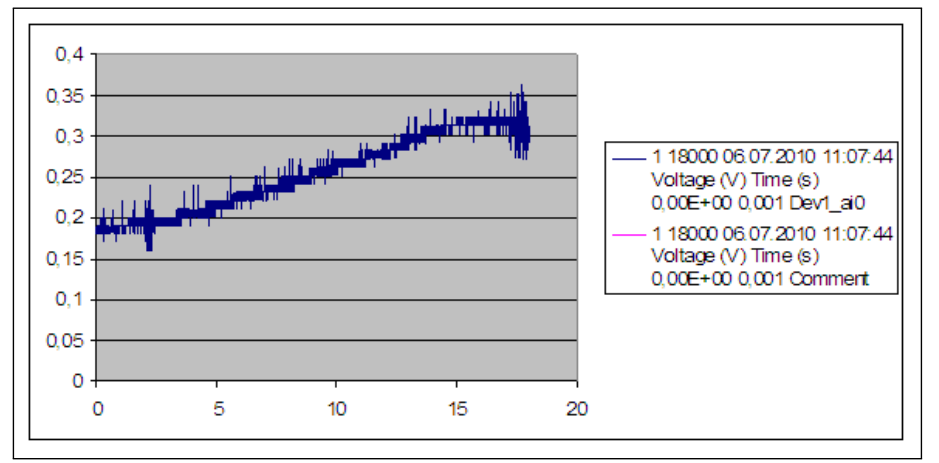

Figure 12:

\section{Conclusions}

The paper treats the control problem of a class of hyper-redundant arms with continuum elements. The observability problems for these models described by partial differential equations are analyzed. The measuring systems are based on the sensors placed on the boundary of the arm. Several observers are proposed for reconstructing the full state of the arm. A back-stepping technique is used in order to design a boundary control algorithm. The numerical simulations and an experimental platform illustrate the effectiveness of the method.

\section{Acknowledgement}

The research presented in this paper was supported by the Romanian National University Research Council CNCSIS through the IDEI Research Grant ID93 and by CNCSIS - UEFISCSU, project number PNII - IDEI code 289/2008.

\section{Appendix 1}

The control algorithm is derived by using the back-stepping method developed in [11]. The coordinate transformation

$$
\mathcal{w}(\mathrm{t}, \mathrm{s})=\tilde{\mathrm{q}}(\mathrm{t}, \mathrm{s})-\int_{0}^{s} \mathrm{k}(\mathrm{s}, z) \tilde{\mathrm{q}}(\mathrm{t}, z) \mathrm{d} z \quad \text { (A.1.1.) }
$$

transforms the error system $(4.6)$ - (4.9) into a stable $(\mathrm{b}<0)$ target system

$$
\begin{gathered}
\ddot{w}=a w_{s s}+b \dot{w} \quad(\text { A.1.2. }) \\
w_{s}(t, 0)=0 \quad(\text { A.1.3. }) \\
w_{s}(t, l)=0 \quad(\text { A.1.4.) } \\
\dot{w}(0, s)=0, s \in[0, l] \quad(\text { A.1.5.) }
\end{gathered}
$$


where

$$
\lim _{t \rightarrow \infty} w(t, s)=0, s \in[0, l] \quad(\text { A.1.6.) }
$$

From (A.1.1) we obtain

$$
\begin{aligned}
& \dot{w}=\dot{\tilde{q}}-\int_{0}^{s} k(s, z) \dot{\tilde{q}}(t, z) d z \quad(\text { A.1.7.) } \\
& \ddot{w}=\ddot{\tilde{q}}-\int_{0}^{s} k(s, z)\left(a \tilde{q}_{s s}+b \dot{\tilde{q}}+c \tilde{q}-k_{1}(z) \tilde{\mathbf{q}}(t, 0)\right) d z \\
& w_{s s}=\tilde{\mathbf{q}}_{s s}-\int_{0}^{s} k_{s s}(s, z) \tilde{\mathbf{q}}(t, z) d z-k_{s}(s, s) \tilde{\mathbf{q}}(t, s)-\frac{d k(s, s)}{d s} \cdot \tilde{\mathbf{q}}(t, s)-k(s, s) \tilde{\mathbf{q}}_{s}(t, s)
\end{aligned}
$$

If we substitute (A.1.7), (A.1.8) in (A.1.2) and integrate by parts we get

$$
\begin{aligned}
& \int_{0}^{s}\left(-a k_{z z}(s, z)+a k_{s s}(s, z)+c k(s, z)\right) \tilde{q}(t, z) d z+ \\
+ & \left(c+a k_{z}(s, s)+a k_{s}(s, s)+a \frac{d k(s, s)}{d s}\right) \tilde{q}(t, s)+ \\
+ & +\left(-a k_{z}(s, 0)-k_{1}(s)-\int_{0}^{s} k_{1}(z) k(s, z) d z\right)=0
\end{aligned}
$$

For left hand side to be zero, the condition (4.13), (4.14) can be easily inferred. From (A.1.7), the velocities at $\mathrm{t}=0, \mathrm{~s}=\mathrm{l}$ will be

$$
\dot{w}=\dot{\tilde{q}}(0, l)+\int_{0}^{l} k(l, z) \dot{\tilde{q}}(0, z) d z
$$

and by the boundary and initial conditions (3.8), (4.9), (A.1.5), we obtain

$$
k(l, z)=0, z \in[0, l]
$$

From (A.1.1) we get

$$
w_{s}(t, s)=\tilde{q}_{s}(t, s)-\int_{0}^{s} k_{s}(s, z) \tilde{q}(t, z) d z-k(s, s) \tilde{q}(t, s)
$$

and by using the boundary condition (4.7) this relation becomes

$$
-k_{0} \tilde{\mathbf{q}}(t, 0)-k(0,0) \tilde{\mathbf{q}}(t, 0)=0
$$

or

$$
k_{0}=-k(0,0) \quad(\text { A.1.14. })
$$

Also, if we consider that the desired position is defined by $q^{\mathrm{d}}(\mathrm{s}), \mathrm{s} \in[0, \mathrm{l}]$ and use the boundary conditions (3.7), (A.1.4) in the relation (A.1.13), the control law (4.12) is easily obtained.

\section{Appendix 2}

The back-stepping transformation is chosen as $[11,12]$ 


$$
w(t, s)=\tilde{\mathbf{q}}(t, s)-\int_{s}^{l} k(s, z) \tilde{\mathbf{q}}(t, z) d z
$$

with the target system defined by (A.1.2) - (A.1.5). By using the same procedure as in Appendix 1, we obtain

$$
\begin{aligned}
& \left(c-a k_{z}(s, s)-a k_{s}(s, s)-a \frac{d k(s, s)}{d s}\right) \tilde{q}(t s)+ \\
& +\left(-k_{1}(s)+\int_{s}^{l} k_{1}(z) k(s, z) d z+a k_{z}(s, l)\right) \tilde{q}(t, l)+ \\
& +\int_{s}^{l}\left(c k(s, z)-a k_{z z}(s, z)+a k_{s s}(s, z)\right) \tilde{q}(t, z) d z=0
\end{aligned}
$$

or

$$
\begin{gathered}
a k_{s s}(s, z)-a k_{z z}(s, z)+c k(s, z)=0 \\
k(s, s)=\frac{c}{2 a} s,(\operatorname{fork}(0,0)=0) \\
k_{1}(s)=a k_{s}(s, l)+\int_{s}^{l} k_{1}(z) k(s, z) d z
\end{gathered}
$$

From (A.2.1) the velocities at $\mathrm{t}=0, \mathrm{~s}=0$ will be

$$
\dot{w}(0,0)=\dot{\tilde{q}}(0,0)+\int_{0}^{l} k(0, z) \dot{\tilde{q}}(0, z) d z
$$

and the boundary conditions (3.8), (4.26), (A.1.5) require

$$
k(0, z)=0
$$

From the back-stepping transformation (A.2.1) we obtain

$$
w_{s}(t, s)=\tilde{q}_{s}(t, s)-\int_{s}^{l} k_{s}(s, z) \tilde{q}(t, z) d z+k(s, s) \tilde{q}(t, s)
$$

By using the boundary conditions for $\mathrm{s}=\mathrm{l},(4.25)$, (A.1.4), it results

$$
-k_{0} \tilde{\mathbf{q}}(t, l)+k(l, l) \tilde{\mathbf{q}}(t, l)=0
$$

or

$$
k_{0}=k(l, l)
$$

From (A.2.8), for $\mathrm{s}=1$ and the condition (4.20), the control law (4.27) is obtained. 


\section{Bibliography}

[1] Hemami, A., Design of light weight flexible robot arm, Robots 8 Conference Proceedings, Detroit, USA, June 1984, pp. 1623-1640.

[2] Gravagne, Ian A., Walker, Ian D., On the kinematics of remotely - actuated continuum robots, Proc. 2000 IEEE Int. Conf. on Robotics and Automation, San Francisco, April 2000, pp. 2544-2550.

[3] Gravagne, Ian A., Walker, Ian D., Kinematic Transformations for Remotely-Actuated Planar Continuum Robots, Proc. 2000 IEEE Int. Conf. on Rob. and Aut., San Francisco, April 2000, pp. 19-26.

[4] Chirikjian, G. S., Burdick, J. W., An obstacle avoidance algorithm for hyper-redundant manipulators, Proc. IEEE Int. Conf. on Robotics and Automation, Cincinnati, Ohio, May 1990, pp. $625-631$.

[5] Mochiyama, H., Kobayashi, H., The shape Jacobian of a manipulator with hyper degrees of freedom, Proc. 1999 IEEE Int. Conf. on Robotics and Automation, Detroit, May 1999, pp. 2837- 2842 .

[6] Robinson, G., Davies, J.B.C., Continuum robots - a state of the art, Proc. 1999 IEEE Int. Conf. on Rob and Aut, Detroit, Michigan, May 1999, pp. 2849-2854.

[7] Ivanescu, M., Stoian, V., A variable structure controller for a tentacle manipulator, Proc. IEEE Int. Conf. on Robotics and Aut., Nagoya, 1995, pp. 3155-3160.

[8] Ivanescu, M., Florescu, M.C., Popescu, N., Popescu, D., Position and Force Control of the Grasping Function for a Hyperredundant Arm, Proc.of IEEE Int. Conf.on Rob. and Aut., Pasadena, California, 2008, pp. 2599-2604.

[9] Ivanescu, M., Bizdoaca, N., Florescu, M., Popescu,N., Popescu, D., Frequency Criteria for the Grasping Control of a Hyper-redundant Robot, Proc.of IEEE International Conference on Robotics and Automation, Anchorage, Alaska (ICRA 2010), May 3 - 8, 2010, pp. 1542-1549.

[10] Miller, D.W., Collins, S.A., Peltzman, S.P., Development of Spatially Convolving Sensors for Structural Control Applications, the 31st AIAA Structures, Structural Dynamic and Materials Conference, 1992, paper 90 - 1127.

[11] Krstic, M., Smyshlyaev, A., Boundary Control of PDEs: A Short Course on Backstepping Design, VCSB, 2006.

[12] Krstic, M., Compensation of Infinite - Dimensional Actuator and sensor Dynamic, IEEE Control Systems, February 2010, vol. 30, no. 1, pp. 22 - 41.

[13] Camarillo, D., Milne, C., Mechanics Modeling of Tendon - Driven Continuum Manipulators, IEEE Trans. On Robotics, vol. 24, no. 6, December 2008, pp. 1262 - 1273. 